\title{
RIEMANN SURFACES AND BOUNDED HOLOMORPHIC FUNCTIONS
}

BY

\author{
WALTER PRANGER
}

\begin{abstract}
The principal result of this article asserts the equivalence of the following four conditions on a hyperbolic Riemann surface $X$ :

(a) the following set $\{z|| f(z)|<\sup | f \mid$ on $K$ for every bounded holomorphic section $f$ of $\xi\}$ is compact for every unitary vector bundle $\xi$ and every compact set $\boldsymbol{K}$;

(b) every unitary line bundle has nontrivial bounded holomorphic sections and the condition in (a) holds for $\xi=i_{d}$;

(c) every unitary line bundle has nontrivial bounded holomorphic sections and $X$ is regular for potential theory;

(d) every unitary line bundle has nontrivial bounded holomorphic sections and $X$ is its own $B$-envelope of holomorphy.

If $X$ is a subset of $\mathbf{C}$, these are also equivalent to the following:

(e) for every unitary line bundle $\xi$ the bounded holomorphic sections are dense in the holomorphic sections.
\end{abstract}

1. Introduction. This work provides results directed toward the problem of identifying a class $\mathfrak{B}$ of complex manifolds which plays the same role for bounded holomorphic functions that Stein manifolds play for holomorphic functions. However, the results here are restricted to one-dimensional manifolds even though certain of them are meaningful to all dimensions.

As the work of $\mathrm{H}$. Widom [12] has shown, the unitary vector bundles and their bounded holomorphic section are fundamental in the study of the Hardy spaces on a Riemann surface and we should expect these objects to be of equal importance in other such situations. Studying the results of [12], we can conjecture that an appropriate class of manifolds $\mathfrak{B}$ will be found by imposing conditions which assure enough bounded holomorphic sections for every unitary line bundle. A requirement which may lead to this end is that the hull, $\hat{K}$, of a set $K$ relative to the set of bounded holomorphic sections of a unitary vector bundle shall be compact whenever $K$ is compact and this is for every such bundle. The reasons for believing that this resolves the problem are developed in this paper.

We refer to [12] for the terminology and more details concerning the facts in this section. $X$ denotes a hyperbolic Riemann surface; $\xi$ denotes a unitary vector bundle over $X$. Each unitary line bundle can be associated with a representation of the first homology group $G$ of $X$; each such line bundle has an inverse $\bar{\xi}$ and a product is defined. Equivalence classes of such line bundles can be identified with $G^{*}$ the dual group of $G$. With each unitary vector bundle $\xi$ we have the space $H(\xi)$

Presented to the Society August 12, 1978; received by the editors December 14, 1978.

AMS (MOS) subject classifications (1970). Primary 30A98; Secondary 30A76.

(C) 1980 American Mathematical Society 0002-9947/80/0000-0252/\$03.00 
of holomorphic sections of $\xi ; H\left(i_{d}\right)=H$ is the space of holomorphic functions. Since the bundles are unitary every section has an absolute value which is a function, so we may define $B(\xi)$ to be the set of those members of $H(\xi)$ which have bounded absolute values; $B\left(i_{d}\right)=B$. The Hardy spaces are $H_{p}(\xi)$ where $0<p<$ $\infty$ and $h$ is a member of $H_{p}(\xi)$ if $h$ is a holomorphic section of $\xi$ and $|h|^{p}$ has a harmonic majorant. One key fact to be used repeatedly is the following. A necessary and sufficient condition that a function on $X$ be the absolute value of a member of some $B(\xi)$ for a unitary line bundle $\xi$ is that it be identically zero or have the form $\exp (c-u-p)$ where $c$ is a nonnegative number, $u$ is a positive harmonic function and $p$ is a discrete Green potential, i.e., $p(z)=\Sigma g\left(z, a_{j}\right)$ where each $a_{j}$ may be repeated a finite number of times and $g(z, a)$ denotes the Green function for $X$ with pole at $a$. This fact is a consequence of the Szegö-Solomentsev theorem of M. Heins [5].

We set $X_{\alpha}=\{z \mid g(z, a)>\alpha\}$ and let $\beta(\alpha, a)$ denote the first Betti number of $X_{\alpha}$.

Widom's THEOREM [12, p. 305]. If $\int_{0}^{\infty} \beta(\alpha, a) d \alpha<\infty$, then every unitary vector bundle $\xi$ has a nontrivial section in $B(\xi)$. If $\int_{0}^{\infty} \beta(\alpha, a) d \alpha=\infty$ then there is a line bundle $\xi$ such that $H_{1}(\xi)$ is trivial.

Trivial means that $B(\xi)$ consists of the zero section only. One of the consequences of this theorem is that if the integral converges for some $a$, then it converges for every $a$. Those hyperbolic domains for which that integral in Widom's theorem converges are referred to as Widom domains in [9].

Not only is there a group structure on the unitary line bundles but the sections corresponding to different bundles may be multiplied. If $\xi$ and $\eta$ are unitary line bundles and $f \in H(\xi), h \in H(\eta)$, then $f h \in H(\xi \eta)$. As a consequence the set $B(\xi)$ are modules over $B$. When $K$ is a subset of $X$ and $A$ is a set of sections of some unitary vector bundle, the $A$-hull of $K$ is defined by

$$
\hat{K}(A)=\{z|| f(z) \mid \leqslant p(K, f) \text { for every } f \in A\},
$$

here $p(K, f)=\sup |f|$ taken over the set $K$. The topology on the spaces $H(\xi)$ is the usual vector space topology defined by the seminorms $p(K, f)$ for $K$ compact.

2. The class $\mathfrak{B}$ and its properties. A hyperbolic Riemann surface is of class $\mathfrak{B}$ if for every unitary vector bundle $\xi$ and for every compact set $K$ the set $\hat{K}(B(\xi))$ is compact. Every surface of class $\mathfrak{B}$ is a Widom domain but the converse is false. The punctured disk is a Widom domain but not of class $\mathscr{B}$ for if $K$ is a circle surrounding the origin, then $\hat{K}(B)$ is not compact.

It is easy to show that for any Widom domain the space $B$ separates points and provides local coordinates. Some other properties of Widom domains and surfaces of class $\mathfrak{B}$ follow.

Proposition 1. If $X$ is of class $\mathfrak{B}$, then $B$ is dense in $H$.

Proof. We make use here of Bishop's generalization of Mergelyan's theorem [2] and apply this result to the algebra $B$. Corollary 2 of [2, p. 48] applied to the compact set $\hat{K}(B)$ yields the result that every function holomorphic on the interior 
of $\hat{K}(B)$ and continuous on $\hat{K}(B)$ is the uniform limit thereon of members of $B$. Now we can exhaust $X$ by a sequence of compact sets $K$ and apply the above reasoning to each of the sets $\hat{K}(B)$; since every $f \in H$ is continuous on $\hat{K}(B)$ and holomorphic on its interior, it follows that $B$ is dense in $H$.

The surface $X$ is regular if for each $\alpha>0$ the set $\{z \mid g(z, a)>\alpha\}$ is relatively compact.

Proposition 2. If $X$ is a Widom domain, then there is a Widom domain $Y$ which is regular, contains $X$ as a subset, and for which the complement of $X$ is a discrete set.

The proof of this result appears in [4, Theorem 3, p. 279].

The space $B$ equipped with the sup norm topology is denoted by $H^{\infty}$.

Proposition 3. If $X$ is a Widom domain, then $X$ is homeomorphic to its image in the spectrum of $H^{\infty}$.

Proof. We suppose first that $X$ is regular. We have the map $i$ from $X$ to the spectrum of $H^{\infty}$ where $i(a)$ is the evaluation at $z=a$. The map is continuous and, because $B$ separates points, $i$ is injective. We must show that the inverse of $i$, restricted to the image of $X$, is continuous. A sequence of evaluations $m_{n}$ converges to an evaluation $m$ if and only if $\lim \left(m_{n}(f)\right)=m(f)$ for each $f \in H^{\infty}$. Suppose that $m_{n}(f)=f\left(a_{n}\right)$ and $m(f)=f(a)$; we want to show that the sequence $\left\{a_{n}\right\}$ converges in $X$ to $a$. This is clear enough when the sequence converges to some point of $X$. Suppose $\left\{a_{n}\right\}$ has no convergent subsequences. Since $X$ is regular it is possible to choose a subsequence of $\left\{a_{n}\right\}$ (denoted again by $a_{n}$ ) with the property that $\sum g\left(a, a_{n}\right)<\infty$. Then $\exp \left(-\sum g\left(z, a_{n}\right)\right)$ is the absolute value of an $f$ which belongs to $B(\xi)$ for some $\xi$. We choose an $h \in B(\bar{\xi})$ so $|h(a)| \neq 0$. Set $F=h f$ so $F \in B, F\left(a_{j}\right)=0$ for each $j$ while $F(a) \neq 0$. Therefore it is not possible that $\lim m_{n}=m$. If $X$ is not regular it is contained in a regular Widom domain $Y$ by Proposition 2 and its complement is discrete. From this it is clear that $X$ and $Y$ have the same spectrum and $X$ is also homeomorphic to its image therein.

Given a Riemann surface for which $B$ separates points we want to consider the largest surface $Y, X \subseteq Y$, with the property that every bounded holomorphic function on $X$ extends to a bounded holomorphic function on $Y$. This $B$-envelope of $X$ exists and is unique up to conformal equivalence, (see [6, pp. 91-96]).

Proposition 4. If $X$ is a Widom domain and is regular, then $X$ is its own $B$-envelope of holomorphy.

Proof. Let $Y$ denote the $B$-envelope of $X$ and let $c$ belong to the boundary of $X$ in $Y$. Since $X$ is regular there is a sequence $\left\{z_{j}\right\}$ in $X$ which converges to $c$ and converges so rapidly that $\Sigma g\left(a, z_{j}\right)=p(a)$ is convergent for some $a$. Then $\exp (-p(z))$ is the absolute value of a section $f$ of some unitary line bundle $\xi$ and $f \in B(\xi)$. We can choose $h \in B(\bar{\xi})$ so $|h(a)| \neq 0$. Then $F=h f \in B$ also extends to $Y$, i.e., extends to the point $c$ and $F \neq 0$. But $F\left(z_{j}\right)=0$ for each $j$ and the sequence $\left\{z_{j}\right\}$ converges to $c$, so $F \neq 0$, a contradiction. Thus no such $c$ exists and $X=Y$. 
Let $A$ denote the closure of $B$ in $H$ and suppose that $X$ is a Widom domain. The results of Bishop [1, p. 508] imply that there is a Riemann surface $Y$ and a uniformly closed subalgebra $A^{\prime}$ of holomorphic functions on $Y$ such that

(a) $X \subseteq Y$,

(b) $A=\left.A^{\prime}\right|_{X}$,

(c) for every compact $K \subseteq Y, \hat{K}\left(A^{\prime}\right)$ is the set $K$ union with those components of its complement which are relatively compact and $\hat{K}\left(A^{\prime}\right)$ is compact.

Proposition 5. If $X$ is a Widom domain and $X$ is its own B-envelope of holomorphy, then $\hat{K}(B)$ is compact for every compact $K$.

Proof. It follows from (b) above that every member of $B$ extends to $Y$. Since the $B$-envelope $X_{\infty}$ of $X$ is maximal with respect to this property, $Y \subseteq X_{\infty}$. Thus $X=Y$. Now (c) above means that $\hat{K}(A)$ is compact for every compact set $K$. But $B$ is dense in $A$ so $\hat{K}(B)$ and $\hat{K}(A)$ are identical.

3. The main theorems. Essentially every Widom domain $X$ can be enlarged to a maximal Widom domain $X_{B}$ and $X_{B}$ is obtained by forming the $B$-envelope of $X$ or equivalently by filling in the punctures of $X$.

THEOREM 1. If $X$ is a hyperbolic Riemann surface, then the following statements are equivalent.

(1) $X$ is of class $\mathfrak{B}$,

(2) $X$ is a Widom domain and for every compact set $K, \hat{K}(B)$ is compact,

(3) $X$ is a Widom domain and regular,

(4) $X$ is a Widom domain and $X$ is its own B-envelope.

Proof. Statement one implies statement two. This is trivial.

LEMMA 1. Suppose that for every $a \in X$ and for every unitary vector bundle $\xi$ there is an $f \in B(\xi)$ with $|f(a)| \neq 0$. Then for every set $K \subseteq X, \hat{K}(B(\xi)) \subseteq \hat{K}(B)$ for every $\xi$.

Proof. If $a \notin \hat{K}(B)$, then a standard argument shows that for every $M>0$ and for every $\varepsilon>0$ there is an $h \in B$ such that $h(a)=M$ and $p(K, h)<\varepsilon$. When $f \in B(\xi), h f \in B(\xi)$. Suppose $a \notin \hat{K}(B)$ but $a \in \hat{K}(B(\xi))$. Then

$$
M|f(a)| \leqslant p(K, h f) \leqslant p(K, h) p(K, f) \leqslant \varepsilon p(K, f) .
$$

Hence for every $f \in B(\xi),|f(a)| \leqslant(\varepsilon / M) p(K, f)$. As $\varepsilon$ and $M$ are arbitrary $|f(a)|=0$ which is the negation of the hypothesis.

In the case when $\xi$ is a unitary line bundle one can show that $\hat{K}(B(\xi))=\hat{K}(B)$ for every $K$ and for every $\xi$.

Statement two implies statement one. If $X$ is a Widom domain, the hypotheses of Lemma 1 hold. If $\hat{K}(B)$ is compact for every compact set $K$, then $\hat{K}(B(\xi)) \subseteq \hat{K}(B)$. As $\hat{K}(B(\xi))$ is closed it is also compact, so $X$ is of class $\mathfrak{B}$.

Statement three implies statement two. Proposition 4 implies that $X$ is its own $B$-envelope of holomorphy so Proposition 5 implies that $\hat{K}(B)$ is compact for every compact $K$. 
Statement one implies statement three. Suppose $X$ is of class $\mathfrak{B}$. The conclusion of Proposition 2 implies there is Widom domain $Y$ which is regular and which contains $X$ as the complement of a discrete set. If $a$ belongs to $Y$ but not to $X$, then a small loop $K$ surrounding the point $a$ is compact but $\hat{K}(B)$ must have $a$ as a limit point, so it is not compact. Thus no such $a$ exists and $X=Y$ and $X$ is regular.

Statement four implies statement two. This is just Proposition 5. The concluding implication is statement three implies statement four. This is merely a restatement of a previous argument. If $X$ is a regular Widom domain and $X \subseteq Y$, there exists a sequence $\left\{z_{j}\right\}$ in $X$ converging to $c \in Y$ and an $F \in B$ which is not identically zero but for which $F\left(z_{j}\right)=0$. No such $F$ can extend to be holomorphic on $Y$. This completes the proof of Theorem 1 .

In case more is known about the surface $X$ another condition equivalent to those of Theorem 1 is available.

THEOREM 2. If $X$ is a hyperbolic subdomain of $\mathbf{C}$, then $X$ is of class $\mathfrak{B}$ if and only if $B(\xi)$ is dense in $H(\xi)$ for every unitary line bundle $\xi$.

If $B(\xi)$ is dense in $H(\xi)$ for each $\xi$, then it follows that $X$ is a Widom domain since $H(\xi)$ has nontrivial members for every $\xi$ and this is valid whether or not $X$ is a subset of $\mathbf{C}$. But then every $B(\xi)$ has nontrivial members, hence so does $H_{1}(\xi)$, so by Widom's theorem $X$ is a Widom domain. To prove that when $X$ is of class $\mathfrak{B}, B(\xi)$ is dense in $H(\xi)$ for each $\xi$ we require two lemmas.

LEMMA 2. If $B$ is dense in $H$ and if for every compact set $K$ and for every unitary line bundle $\xi$ there is an $h \in B(\xi)$ with no zeros on $K$, then $B(\xi)$ is dense in $H(\xi)$ for every unitary line bundle $\xi$.

Proof. Let $K$ and $\xi$ be given. We can assume with no loss of generality that $K=\hat{K}(B)$ since $\hat{K}(B)$ is itself compact. Let $F \in H(\xi)$ and suppose that $h \in B(\xi)$ has no zeros on $K$. Since $X$ is a Stein manifold and since $K=\hat{K}(B)=\hat{K}(H)$ the holomorphic function $F / h$ can be approximated on $K$ by members of $H$ [7, p. 239]. As $B$ is dense in $H, F / h$ can be approximated on $K$ by members of $B$. Hence for each $\varepsilon>0$ there is an $f \in B$ such that $|F / h-f|<\varepsilon$ on $K$. Then $|F-h f|<$ $\varepsilon p(K, h)$ holds on $K$. As $h f \in B(\xi)$ the conclusion of the lemma follows.

Lemma 3. Suppose that $X \subset \mathbf{C}$ is a Widom domain. For every compact set $K \subseteq X$ and for every unitary line bundle $\xi$ on $X$ there is an $h \in B(\xi)$ which has no zeros on $K$.

Proof. This is taken directly from p. 75 of [13]. Let $f \in B(\xi),|f| \leqslant 1, f$ not identically zero. Let $a_{1}, \ldots, a_{n}$ denote the zeros of $f$ on $K$. Let $a \in X, a \notin K$ and put $k(z)=(z-a)^{n} /\left(z-a_{1}\right) \cdots\left(z-a_{n}\right)$. Then $k f \in B(\xi)$ and has no zeros on $K$.

To complete the proof of Theorem 2 let $X$ be a subdomain of $\mathbf{C}$ which is of class $\mathfrak{B}$. By the result of Proposition $1, B$ is dense in $H$. Now Lemmas 2 and 3 together imply that $B(\xi)$ is dense in $H(\xi)$ for every $\xi$.

The sole impediment to proving Theorem 2 in general is the assertion of Lemma 3 when $X$ is not a subdomain of $\mathbf{C}$. If $X$ is a Widom domain, then for each point $a$ 
and for each $\xi$ there is an $h$ in $B(\xi),|h(a)| \neq 0$; so there is a neighborhood of $a$ on which $h$ is never zero. But to extend from the local condition to the same assertion for any compact set seems difficult. Despite this Theorem 2 is probably true without the restriction to subsets of $\mathbf{C}$.

The next theorem shows that surfaces of class $\mathfrak{B}$ are maximal with respect to each of the classes $B(\xi)$.

Theorem 3. Suppose $X$ is a Widom domain. Suppose $W$ is a Widom domain with the following properties:

(a) $X \subseteq W$,

(b) for some unitary line bundle $\xi$ on $W$ every bounded holomorphic section of the restriction of $\xi$ to $X$ extends to a bounded holomorphic section of $\xi$ on $W$.

Then $W \subseteq X_{B}$.

Proof. We claim first that every bounded holomorphic function on $X$ extends to be holomorphic on $W$. For let $b$ denote such a function on $X$. Let $a \in W$ and let $\xi$ denote a unitary line bundle as in (b) above. Let $F \in B(\xi)$ with $|F(a)| \neq 0$. Then $b F=h$ is a bounded holomorphic section of the restriction of $\xi$ to $X$ so extends to $W$. Hence near the point $a, b=h / F$ is holomorphic. Since $W$ is connected, $b$ can be continued analytically to each point of $W$. Now we consider $W_{B}$ the $B$-envelope of $W$ and claim that $X$ is dense in $W_{B}$. For if not, there is an open connected set $Y \subseteq W_{B}$ for which $Y \cap X=\varnothing$. Let $a \in Y$. As $W_{B}$ is regular there is an $\alpha>0$ such that the set $\{z \mid g(a, z)>\alpha\} \quad\left(g(a, z)\right.$ is the Green function for $\left.W_{B}\right)$ is contained in $Y$. There is a section $F_{a}$ corresponding to some $\eta$ such that $\left|F_{a}\right|=$ $\exp (-g(a)$,$) . We can choose an h \in B(\bar{\eta})$ on $W_{B}$ so $|h(a)| \neq 0$. On the complement of $Y$, which contains $X, k=h / F_{a}$ is a bounded holomorphic function. So $k$ extends to be holomorphic on $W$. But $k$ has a pole at $z=a$ so $a \notin W$ and this is true for every $a \in Y$. This is not possible as $W_{B}-W$ is discrete. So $X$ is dense in $W_{B}$. Now every bounded holomorphic function on $X$ extends to be a bounded holomorphic function on $W$. Therefore $W$ is contained in the $B$-envelope of $X$.

4. Example and counterexamples. The punctured disk is an example of a surface which is a Widom domain but for which $B(\xi)$ is not dense in $H(\xi)$ for any unitary line bundle $\xi$. Given $\xi$ there is a number $t, 0 \leqslant t<1$, such that $z^{t} \in B(\xi)$; in fact every member of $B(\xi)$ has the form $z^{t} b(z)$ for some $b \in B$ [12, p. 312]. Every member of $H(\xi)$ has the form $z^{t} h(z)$ for some $h \in H$. Now we can see that $B(\xi)$ is dense in $H(\xi)$ for some $\xi$ if and only if $B$ is dense in $H$.

There is a surface for which $B$ is dense in $H$ but which is not a Widom domain. It suffices to find a surface for which $B$ is dense in $H$ but which is not regular; such a surface cannot be a Widom domain since it would have to be of class $\mathfrak{B}$ hence be regular. Also, such a surface is its own $B$-envelope. To find such a surface we use the $\Delta$ domains defined in [14]. From the punctured disk we remove a sequence of disks clustering at the origin, $\left|z-x_{n}\right| \leqslant r_{n}, n=1,2, \ldots$, where $\Sigma r_{n} / x_{n}<\infty$. Wiener's criterion [11, p. 104] shows that the origin is not a regular point. For any $\Delta$ domain $X, B$ is dense in $H$. This is easily seen as follows. Runge's theorem shows that for any compact set $K \subseteq X$, any $f \in H$ can be approximated uniformly on $K$ 
using rational functions whose poles lie in the excised disks $\left|z-x_{n}\right|<r_{n}$, at the origin and outside $|z| \leqslant 1$. On the other hand, every function of the form $z^{-s}$, $s=1,2, \ldots$, can be approximated uniformly on $K$ by functions of the form $(z-a)^{-s}$ where $a$ is in an excised disk. Hence every $f \in H$ can be approximated uniformly on $K$ by rational functions whose poles are in the excised disks or outside $|z| \leqslant 1$. The restriction of any such function to $X$ is bounded.

The interior of any compact bordered surface is of class $\mathfrak{B}$. The simplest way to see this is to know first that any such surface is a Widom domain [12, p. 307]. Since it is also regular it is also of class $\mathfrak{B}$. It is also the case that for such surfaces $B(\xi)$ is dense in $H(\xi)$ for every unitary vector bundle $\xi$.

Several authors have considered classes of surfaces which include the Widom domains. In [3] and [4] Hasumi considers surfaces of type $(B)$ and in [8] Neville considers admissible surfaces. In each case the author assumes the existence of an outer section in $B(\xi)$ for each unitary line bundle $\xi$. This means that there is for each $\xi$ an $f \in B(\xi)$ such that $|f|=\exp (-u)$ where $u$ is a quasibounded harmonic function. The existence of outer sections in each $B(\xi)$ implies that the surface is of class $\mathfrak{B}$ as the next proposition shows. From this result it follows that surfaces of type $(B)$ and admissible surfaces are of class $\mathfrak{B}$.

PROPOSITION 6. If the hyperbolic surface $X$ has the property that for every unitary line bundle $\xi, B(\xi)$ has an outer member, then $X$ is of class $\mathfrak{B}$.

Proof. Since every $B(\xi)$ is nontrivial, $X$ is a Widom domain. Let $Y$ denote the $B$-envelope of $X$. Every quasibounded harmonic function on $X$ extends to a harmonic function on $Y$. Let $a \in Y, a \notin X$ and consider the section $f,|f|=$ $\exp \left(-t g_{a}\right)$ where $g_{a}$ is the Green function for $Y$ with pole at $a$ and where $0<t<1$. There is a unitary line bundle $\xi$ on $X$ for which $f \in B(\xi)$. We choose an $h \in B(\bar{\xi})$ so that $h$ is outer and $|h| \leqslant 1$. Then $F=f h$ is a bounded holomorphic function on $X$, hence extends to $Y$. Also, $|F| \leqslant 1$ on $Y$ and $F(a)=0$. Therefore, $|F| \leqslant$ $\exp \left(-g_{a}\right)$ so $|h| \leqslant \exp \left(-(1-t) g_{a}\right)$ and consequently $u \geqslant(1-t) g_{a}$ on $Y$. This is impossible as $u$ is harmonic at $z=a$. Thus no such an $a$ exists and $X=Y$.

In [8, p. 67] it is observed that the interior of any compact bordered surface is an admissible surface. Since admissible surfaces are of class $\mathfrak{B}$ this is another way of seeing that such surfaces are of class $\mathfrak{B}$.

In [13] Widom studied the function $m_{\infty}(\xi, a)=\sup |f(a)|$ where $f \in B(\xi)$ and $|f|<1$. If we put the weak topology on the dual group $G^{*}$, then we may speak of the continuity of $m_{\infty}$ as a function of $\xi$ as is done in [13]. In [10] it is shown that for any Widom domain for which $m_{\infty}$ is continuous in $\xi, B(\xi)$ is dense in $H(\xi)$ for every unitary line bundle $\xi$. Thus any surface for which $m_{\infty}$ is continuous is of class $\mathfrak{B}$. A construction for obtaining surfaces for which $m_{\infty}$ is continuous is given in [13].

5. Concluding remarks. Any surface which is "good" for bounded holomorphic functions should possess the property that $B(\xi)$ is dense in $H(\xi)$ for each unitary vector bundle $\xi$. Hence the first question which should be answered is-does Theorem 2 hold in the general case? Besides this there are other properties which one might expect. 
(1) The set $H^{\infty}$ is dense in the Hardy spaces $H_{p}$ and in the Smirnov class $N^{+}$. When $m_{\infty}$ is continuous these properties hold [13].

(2) Every positive bounded divisor is the divisor of a member of $B$. This refers to the following. A neccessary condition that a positive divisor $\left(z_{j}\right)$, where each $z_{j}$ may be repeated a finite number of times, be the divisor of a bounded holomorphic function is that $\Sigma g\left(a, z_{j}\right)<\infty$ for some $a$. Is this condition also sufficient when $X$ is of class $\mathfrak{B}$ ?

(3) Every unitary line bundle is determined by a bounded divisor. By this we mean that if $\xi$ is a unitary line bundle, there is some divisor $\left(z_{j}\right)$ such that $\Sigma g\left(a, z_{j}\right)<\infty$ for some $a$ and there is an $f \in B(\xi)$ such that $|f(z)|=\exp \left(-\sum g\left(z, z_{j}\right)\right)$. This is analogous to the fact that every vector bundle is determined by a divisor.

\section{REFERENCES}

1. E. Bishop, Analyticity in certain Banach algebras, Trans. Amer. Math. Soc. 102 (1962), 507-544.

2. __ Subalgebras of functions on a Riemann surface, Pacific J. Math. 8 (1958), 29-50.

3. M. Hasumi, Invariant subspaces on open Riemann surfaces, Ann. Inst. Fourier (Grenoble) 24 (1974), 241-286.

4. __ Invariant subspaces on open Riemann surfaces. II, Ann. Inst. Fourier (Grenoble) 26 (1976), 273-299.

5. M. Heins, Hardy classes on Riemann surfaces, Lecture Notes in Math., vol. 98, Springer-Verlag, New York and Berlin, 1969.

6. R. Narasimhan, Several complex variables, Univ. of Chicago Press, Chicago, Ill., 1971.

7. Analysis on real and complex manifolds, North-Holland, Amsterdam, 1973.

8. C. Neville, Invariant subspaces of Hardy classes on ininitely connected open surfaces, Mem. Amer. Math. Soc., No. 160 (1975).

9. Ch. Pommerenke, On the Green's function of Fuchsian groups, Ann. Acad. Sci. Fenn. Ser. AI 2 (1976), 409-427.

10. W. Pranger, Bounded sections on a Riemann surface, Proc. Amer. Math. Soc. 69 (1978), 77-80.

11. M. Tsuji, Potential theory in modern function theory, Maruzen, Tokyo, 1959.

12. H. Widom, $H_{p}$ sections of vector bundles over Riemann surfaces, Ann. of Math. (2) 94 (1971), 304-324.

13. __ The maximum principle for multivalued analytic functions, Acta Math. 126 (1971), 63-82.

14. L. Zalcman, Bounded analytic functions on domains of infinite connectivity, Trans. Amer. Math. Soc. 144 (1969), 241-269.

Department of Mathematical Sciences, DePaul University, Chicago, Illinois 60614 